\title{
Primary Squamous Cell Carcinoma of the Rectum: a Case Report
}

Faidzal $\mathrm{O}^{\mathrm{a}}$, Azmi MNa, Kalavathi R

a Department of Surgery, Kulliyah of Medicine, International Islamic University, Malaysia

${ }^{b}$ Department of Pathology, Hospital Selayang, Selangor, Malaysia

ABSTRACT

Squamous cell carcinoma of the rectum is a very rare malignancy. In the literature, the incidence has been reported as 0.25 to 0.1 per 10000 colorectal carcinomas. Due to its rarity, the treatment and behavior of this tumor have only been discussed in case reports. As a result, several theories regarding their pathogenesis have been proposed. We will describe our experience in managing this rare malignancy in a 69-year-old man who presented with altered bowel habit. Surgery remains the mainstay of treatment. Issues regarding additional treatment have been presented in the literature. The rarity of this tumour also raises issues regarding criteria for diagnosis which we will try to highlight and discuss.

KEYWORDS: Squamous cell carcinoma, rectum

\section{INTRODUCTION}

Colorectal carcinoma is among the top ten most common malignancies in Malaysia. It accounts for $14.2 \%$ of male cancers making it the commonest cancer among men and the third most common cancer among women $\left(10.1 \%\right.$ of female cancers). ${ }^{1}$ More than 90 percent of these cases are adenocarcinomas. Pure squamous-cell carcinoma (SCC) is not uncommon in tumours, which develop in glandular organs such as uterus, lung, and pancreas but is a rare in the intestinal tract. Squamous cell carcinomas of the colon and rectum represent a very rare malignancy. ${ }^{2}$ We present our experience in managing this rare tumor and highlight the proposed etiology and criteria for diagnosis.

\section{CASE REPORT}

A 68-year-old man was referred to our unit complaining of change in bowel habits during the preceding six months. Colonoscopy revealed an obstructing mass at the upper rectum $(10 \mathrm{~cm}$ above the dentate line). Initial pathology demonstrated a moderately

Corresponding author:

Professor Dr Azmi Md Nor

Consultant Colorectal Surgeon and

Head of Department

Department of Surgery

Kulliyah of Medicine

International Islamic University Malaysia

Jalan Hospital, 251500 Kuantan

Pahang, Malaysia

Tel: (6)09-5706160

Fax: (6)09-514 6090

Email: azmimn@iium.edu.my differentiated squamous cell carcinoma of the rectum. No evidence of metastatic disease was identified on abdominal and thoracic CT scan.

An anterior resection of the rectum was performed. Microscopic examination demonstrated a squamous cell carcinoma of the rectum invading the serosa and adjacent omentum (Figure 1 and 2). Two out of 42 lymph nodes were infiltrated by tumour cells.

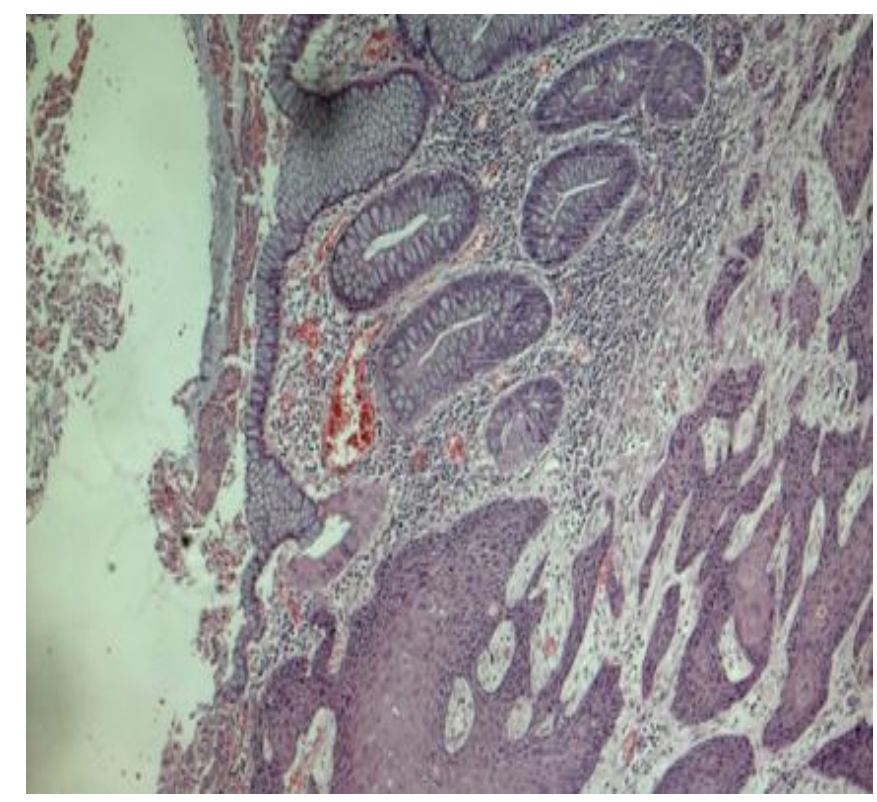

Figure 1. Squamous cell carcinoma arising from rectal mucosa $(\mathrm{X} 2)$ 


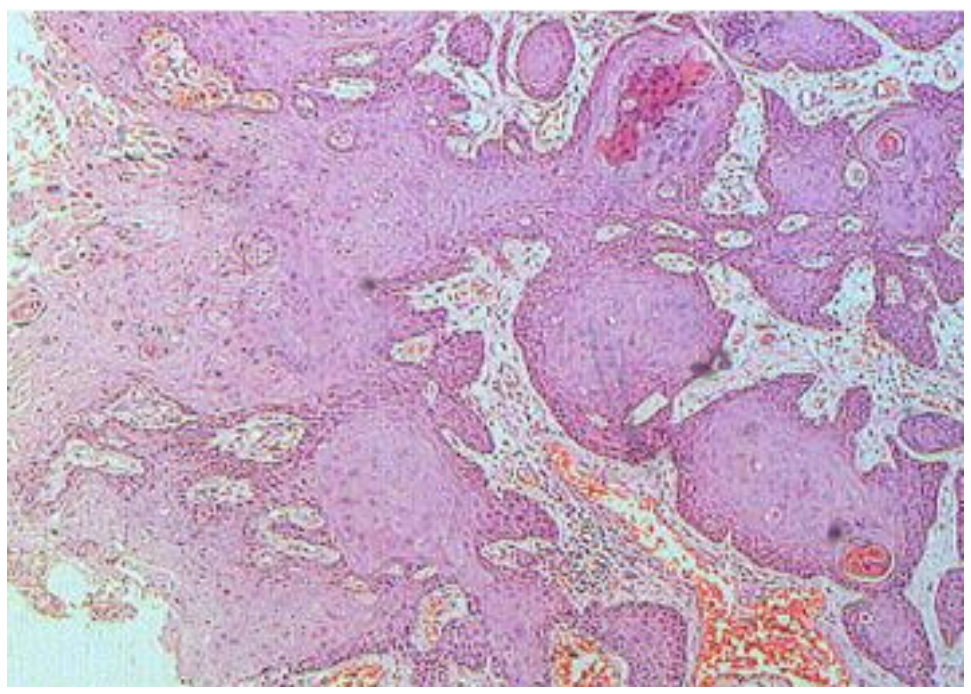

Figure 2. Well differentiated squamous cell carcinoma arranged as irregular sheets and masses with keratin pearls (X5)

Postoperative course was uneventful. Adjuvant chemotherapy with Folinic acid $\left(20 \mathrm{mg} / \mathrm{m}^{2} /\right.$ day on days $1-4)$, 5 -fluorouracil $\left(400 \mathrm{mg} / \mathrm{m}^{2} /\right.$ day on days $\left.1-5\right)$ was given for six cycles at four weekly. The pelvis was given radiotherapy $50 \mathrm{~Gy}$ in 25 fractions for five weeks. The patient tolerated the treatment well, and the review at 15 months found him to be well and disease free.

\section{DISCUSSION}

Squamous cell carcinomas of the rectum are extremely rare. It was first described in 1904 in a patient with an adenosquamous carcinoma of the caecum. In 1909, another case of adenosquamous carcinoma of the sigmoid colon was reported. Ten years later in 1919 the first case of a pure squamous cell colon carcinoma was then reported. Their incidence has been reported to be $0.25-1 / 10000$ of colorectal neoplasms. ${ }^{2}$

Certain criteria must be fulfilled before a diagnosis of primary squamous-cell carcinoma of the rectum is made: ${ }^{2}$

1. Metastases from other organs to the bowel must be ruled out

2. A squamous-lined fistulous tract must not involve the affected bowel.

3. Squamous cell carcinomas of the anus with proximal extension must be excluded (lack of continuity between the tumour and the normal squamous epithelium).

4. Squamous cell carcinoma must be confirmed by histologic analysis.

The actual pathogenesis is poorly understood due to the rarity of condition. Several theories were proposed by Anagnostopoulus ${ }^{2}$ in his recent review to explain the origin of these tumours:

1. Theory of a pluripotent stem cell capable of multidirectional differentiation. The majority of pathologic reports describe squamous cell carcinomas arising within poorly differentiated tumours with an admixture of squamous, glandular and neuroendocrine features. Similar evidence for multidirectional differentiation has been reported in other anatomic sites, including the lung, oesophagus, endometrium, and other sites of the gastrointestinal tract.

2. Proliferation of uncommitted basal cells into squamous cells, which undergo malignant change. Recurrent damage to the glandular epithelium stimulates proliferation of uncommitted or reserve cells, and the resultant tissue may be benign or malignant.

3. Squamous metaplasia of the glandular epithelium resulting from chronic irritation Conditions such as ulcerative colitis, radiation exposure, or human papilloma virus may allow squamous metaplasia and development of these tumours. True squamous metaplasia associated with an extensive mucosal squamous differentiation has rarely been reported.

4. Squamous differentiation of adenomas and adenocarcinomas. This explanation was proposed based upon the finding of areas of squamous differentiation in three of 750 adenomas $(0.4 \%)$. Similar evaluation of 75 additional adenomas with invasive adenocarcinoma revealed a single adenoma with focus of invasive squamous cell carcinoma.

Clinical presentation is similar to colon adenocarcinoma (change in bowel habits, rectal bleeding, abdominal pain, weight loss, anorexia). ${ }^{3}$ The rectum and the proximal colon are the most frequently involved sites. We recognized the rarity of this condition, which makes their natural history less defined. Frizelle et al. concluded that the prognosis is similar to colorectal adenocarcinoma for Stage I to II node-negative disease. Nodal involvement signifies a worse prognosis than for similar stage adenocarcinoma. ${ }^{4}$ Mixed Squamous cell carcinomas with small cell or undifferentiated features are associated with a particularly poor prognosis. Other features predictinga poor prognosis include right sided lesions, ulcerated or annular carcinomas, grade 3 or 4 cancer and Stage IV 
disease. ${ }^{4}$ Surgical resection is the most appropriate and effective treatment for squamous cell colorectal carcinomas. The type of procedure depends upon the location of the tumour and the presence or absence of coexisting colorectal disease. The role of adjuvant chemotherapy and radiotherapy remains undefined. ${ }^{2}$ Combined chemoradiotherapy with 5 -FU based chemotherapy and fractionated radiotherapy as a primary treatment for this condition has been reported. However, due to the rarity of the disease and hence the small number treated, the author suggested further evaluation of the treatment regime. ${ }^{5}$

In conclusion, squamous cell carcinoma of the colon and rectum are very rare neoplasms. The diagnosis should be made only after the criteria are met. In terms of treatment, surgery remains the mainstay in the management of these tumours. Small numbers and hence limited resources from the literature make it difficult to standardize treatment. Adjuvant chemotherapy and radiotherapy may have a role, especially for node-positive patients.

\section{ACKNOWLEDGEMENT}

The authors would also like to thank the assistance given by Professor Dr Naznin Muhamad and Associate Professor Dr Norlelawati A. Talib (Dept. of Basic Sciences, Kulliyah of Medicine, IIUM), and Dr Nora Harun (Dept. of Pathology, Hospital Tengku Ampuan Afzan) for the preparation of histopathology slides.

\section{REFERENCES}

1. Lim GCC, Halimah Y. Second Report of the The National Cancer Registry, Cancer incidence in Malaysia 2003. National Cancer Registry: Kuala Lumpur, 2004.

2. Anagnostopoulos $G$, Sakorafas $G H$, Kostopoulos $P$, et al. Squamous cell carcinoma of the rectum: a case report and review of the literature. Eur $\mathrm{J}$ Cancer Care 2005; 14:70-4.

3. Gelas $T$, Peyrat P, Francois $Y$, et al. Primary squamous-cell carcinoma of the rectum: report of six cases and review of the literature. Dis Colon Rectum 2002; 45:1535-40.

4. Frizelle FA, Hobday KS, Batts KP, Nelson H. Adenosquamous and squamous carcinoma of the colon and upper rectum: a clinical and histopathologic study. Dis Colon Rectum 2001; 44:341-6.

5. Yeh J, Hastings J, Rao A, Abbas MA. Squamous cell carcinoma of the rectum: a single institution experience. Tech Coloproctol 2012; 16:349-54. 